\title{
PATH INTEGRALS WITH TOPOLOGICAL CONSTRAINTS: AHARONOV-BOHM EFFECT AND POLYMER ENTANGLEMENTS
}

\author{
F.W. WIEGEL \\ Department of Applied Physics, Twente University of Technology, P.O. Box 217, 7500 AE \\ Enschede, The Netherlands
}

Received 24 April 1981

\begin{abstract}
For Wiener- and Feynman integrals over paths with certain topological properties we compare various methods for explicit calculation. This leads to a one-to-one correspondence between the Aharonov-Bohm effect and a certain polymer entanglement problem. We briefly comment on two generalizations of the Aharonov-Bohm effect. First, we consider this effect due to a closed magnetic flux loop of arbitrary shape; next, we consider the combined effect due to a gas of microscopic magnetic flux loops.
\end{abstract}

\section{Introduction}

In recent years theoretical physics has been confronted with some problems which are of a topological nature. These problems arose for the first time in quantum mechanics in the context of the Aharonov-Bohm effect, and later in the study of entangled polymers. They crop up also in molecular biology. In this note we show that a profound relation exists between the topological problems in quantum- and polymer physics and we develop the path-integral formalism that describes them both in a natural way.

The Aharonov-Bohm effect demonstrates that the magnetic vector potential can have observable consequences for an electron that passes through a region in space in which the magnetic field vanishes. The literature on this surprising effect has been reviewed in detail by $\mathrm{Kobe}^{1}$ ). In the usual geometry one considers an infinitely long and infinitesimally thin, straight cylinder which contains a magnetic flux $\Phi$. Electrons pass on either side of this cylinder. When a screen is placed a large distance behind the cylinder one observes an interference pattern, unless

$$
\frac{e \Phi}{h c}=0, \pm 1, \pm 2, \ldots,
$$

where $e<0$ denotes the electron's charge, $h$ Planck's constant and $c$ the speed of light. When this equality does not hold an interference pattern is observed on the screen. This pattern is caused by the presence of a vector potential in that part of space where the electrons move. Note that the 
magnetic field is zero in this part of space; hence no Lorentz force acts on the electron.

At first glance it is not obvious that the Aharonov-Bohm effect is caused by the topology of the electron paths. This becomes clear only when this problem is formulated in the language of path integrals, as was done for the first time by Gerry and Singh ${ }^{2}$ ). In section 2 we shall consider this effect in a more general geometry in which the magnetic flux is confined to some arbitrary, infinitesimally thin, closed, fixed curve $D$ in space, and we shall allow the presence of non-magnetic forces between the electron and this curve. It will be shown for this general case that the Aharonov-Bohm effect is caused by the coincidence of two facts: (a) The curve $D$ divides the collection of electron paths into an infinite number of topologically inequivalent classes; (b) Because of the way in which the vector potential enters into the Lagrangian for the electron the contributions of the different classes to the Green function differ in phase, unless condition (1.1) is fulfilled.

The topological aspect is more immediately obvious in the problem of polymer entanglements. For example, the elastic properties of a closed ring-shaped polymer can differ considerably from those of a linear polymer. This difference is caused by the fact that the configurations of a closed polymer can be divided into a large number of topologically inequivalent classes. In the following pages we shall specifically consider two problems: (a) A closed polymer is entangled with some arbitrary, closed, fixed curve $D$ in space; (b) A closed polymer is entangled with itself. In both cases one is especially interested in calculating the thermodynamic functions of the polymer, and the apriori probability of the different topological classes. For a general review of polymer entanglement calculations the reader is referred to two papers by the author ${ }^{3,4}$ ).

At the beginning of the introduction we noted that topological problems crop up also in molecular biology. Although we shall not discuss these problems thoroughly in this paper we will comment briefly on them now. Topological problems which arise in connection with the conformation and replication of DNA have been studied especially by Fuller ${ }^{5,6}$ ), Crick $^{7}$ ) and Pohl and Roberts ${ }^{8}$ ). Consider a piece of double-stranded DNA. Following Crick we can represent this molecule by a ribbon, where the edges of the ribbon are the centers of the two strands. There is also a line along the center of the ribbon; this will be called the axis of the ribbon. For biochemical reasons one needs to consider only closed ribbons which join back on themselves in such a way that each edge joins only with itself. This excludes, for example, a Möbius strip. There are now three distinct concepts which describe the topology of this circular, double-stranded DNA molecule; (a) the linking number $L$; (b) the twist $T$; (c) the writhing number $W$. The first two of 
these are properties of the ribbon, but the last one is a property only of the axis of the ribbon. Moreover, these three concepts turn out to be related by the simple relation $W=L-T$. We shall not pursue these matters any further, but merely state that the linking number $L$ plays a role both in the AharonovBohm effect and in the theory of polymer entanglements.

\section{The Aharonov-Bohm effect}

In this section we calculate the propagator of an electron in an external field which consists of a magnetic field $\boldsymbol{H}(\boldsymbol{r})$, with vector potential $\boldsymbol{A}(\boldsymbol{r})$, to which some other conservative field with scalar potential $V(r)$ is added. The Lagrangian is given by the expression (cf. ref. 9)

$$
\mathscr{L}=\frac{1}{2} m \dot{\boldsymbol{r}}^{2}+\frac{e}{c} \dot{\boldsymbol{r}} \cdot \boldsymbol{A}-\boldsymbol{V},
$$

where $m$ denotes the electron mass. The propagator between space-time point (i) $\equiv\left(r_{i}, t_{j}\right)$ and $(f) \equiv\left(r_{f}, t_{f}\right)$ is given by the Feynman path integral (cf. ref. 10)

$$
G(\mathrm{f} \mid \mathbf{i})=\int_{(\mathrm{i})}^{(f)} \exp \left(\frac{\mathrm{i}}{\hbar} \int_{t_{\mathrm{i}}}^{t_{t}} \mathscr{L}[\boldsymbol{r}(t)] \mathrm{d} t\right) \mathrm{d}[\boldsymbol{r}(t)],
$$

where the integration is performed over all electron paths $r(t)$ which connect (i) and (f) in space-time. We are interested in the Aharonov-Bohm effect due to a magnetic flux $\Phi$ confined to an infinitesimally thin solenoid the axis of which has the form of a closed continuous directed curve $D$. The vector potential is given by

$$
\boldsymbol{A}(\boldsymbol{r})=\frac{\Phi}{4 \pi} \oint_{D}|r-s|^{-3} \mathrm{~d} s \times(\boldsymbol{r}-s),
$$

where $\mathrm{d} s$ denotes the line element of $D$. Now consider an arbitrary electron path $r(t)$ in the path integral (2.2). Substituting (2.1) into (2.2) the term with the vector potential leads to a term in the exponential

$$
\int_{t_{\mathrm{i}}}^{t_{\mathrm{f}}} \dot{\boldsymbol{r}} \cdot \boldsymbol{A} \mathrm{d} t=\int_{C} \boldsymbol{A} \cdot \mathrm{d} \boldsymbol{r},
$$

where the line integral extends along the contour $C$ traced out by the electron in space. This line integral has some very simple properties which can be found as follows: With the specific choice (2.3) $\operatorname{div} \boldsymbol{A}=0$ everywhere in 
space; curl $\boldsymbol{A}=0$ for $\boldsymbol{r} \notin D$. On $D$ the curl of the vector potential diverges in such a way that

$$
\oint \boldsymbol{A} \cdot \mathrm{d} \boldsymbol{r}=\Phi
$$

for every contour which winds around a line element of $D$ once, in a direction which is connected to the vector representing the line element by the Maxwell right-hand rule. Hence, (2.4) can be written in the form

$$
\int_{C} \boldsymbol{A} \cdot \mathrm{d} \boldsymbol{r}=\int_{C_{0}} \boldsymbol{A} \cdot \mathrm{d} \boldsymbol{r}+n \Phi,
$$

where $C_{0}$ denotes some standard contour-for example, some curve which connects $r_{\mathrm{i}}$ with $\boldsymbol{r}_{\mathrm{f}}$ and which does not pass through $D$-and where the integer $n$ equals the number of times that the closed loop $C-C_{0}$ winds through $D$. Combining the last relation with $(2.1,2)$ one finds for the propagator the expression

$$
G(\mathrm{f} \mid \mathrm{i})=\exp \left(\frac{\mathrm{i} e}{\hbar c} \int_{c_{0}} A \cdot \mathrm{d} r\right) \sum_{n=-\infty}^{+\infty} F_{n}(\mathrm{f} \mid \mathrm{i}) \exp \left(\frac{\mathrm{i} e \Phi}{\hbar c} n\right)
$$

in which $F_{n}$ is defined as the constrained Feyman integral

$$
F_{n}(\mathrm{f} \mid \mathrm{i})=\int_{(\mathrm{i})}^{(\mathrm{f})} \exp \left\{\frac{\mathrm{i}}{\hbar} \int_{\mathrm{i}_{\mathrm{i}}}^{t_{\mathrm{f}}}\left(\frac{1}{2} m \dot{r}-V\right) \mathrm{d} t\right\} \mathrm{d}_{n}[\boldsymbol{r}(t)],
$$

which has to be extended over all trajectories which, when completed into a closed loop by adding $-C_{0}$, wind exactly $n$ times through $D$.

The result (2.7) confirms some ideas which were formulated a decade ago by Schulman') and generalizes them to the Aharonov-Bohm effect of a curved solenoid of arbitrary shape, in the presence of other, conservative forces. If the quantization condition (1.1) holds the phase factors in (2.7a) all equal unity and the summation over $n$ leads to

$$
G(\mathrm{f} \mid \mathrm{i})=F(\mathrm{f} \mid \mathrm{i}) \exp \left(\frac{\mathrm{i} e}{\hbar c} \int_{C_{0}} A \cdot \mathrm{d} r\right)
$$

where $F$ denotes the full, unconstrained, propagator for an electron in the scalar potential $V$, in the absence of the magnetic vector potential. Hence, in this case $G$ will not show an Aharonov-Bohm effect. Of course, an interference pattern might still appear due to the general phase factor 
$\exp \left[(i e / \hbar c) \int_{C_{0}} A \cdot d r\right]$ or as a result of electron scattering from the curve $D$ under the influence of the scalar potential $V$. Such an interference pattern, however, is not an Aharonov-Bohm effect.

\section{Calculational techniques for constrained path integrals}

From a practical point of view the results of the preceeding section seem to provide little assistance in calculating Aharonov-Bohm interference patterns. In this section we discuss several different techniques to calculate topologically constrained path integrals. These techniques will also play a role in the theory of polymer entanglements.

First, one can try to solve the propagator directly from the Schrödinger equation. This would amount to solving the eigenvalue problem

$$
\begin{aligned}
& \mathscr{H} \psi_{k}=E_{k} \psi_{k}, \\
& \mathscr{H}=\frac{1}{2 m}\left(\frac{\hbar}{\mathrm{i}} \nabla-\frac{e}{c} \boldsymbol{A}\right)^{2}+V,
\end{aligned}
$$

and to expand the propagator as a sum over the orthonormal eigenfunctions

$$
G(\mathrm{f} \mid \mathrm{i})=\sum_{k} \psi_{k}(\mathrm{f}) \psi_{k}^{*}(\mathrm{i}) \exp \left(\frac{E_{k} t}{\mathrm{i} \hbar}\right) .
$$

If this sum can be evaluated explicitly the result can be expanded in integer powers of the phase factor $\exp (i e \Phi / \hbar c)$. A glance at (2.7) shows that the coefficient of the $n$th power of this factor equals the Feynman path integral over the $n$-times entangled electron paths, times a trivial phase factor $\exp \left[(i e / \hbar c) \int_{C_{0}} \boldsymbol{A} \cdot \mathrm{d} r\right]$. A calculation of this type seems to be implied in footnote 1 of a recent letter by Bernido and Inomata ${ }^{12}$ ).

Second, one can try to evaluate the constrained path integral directly as follows: Let $S$ denote a surface of which $D$ is the boundary. Orient this surface in such a way that the outward normal vector is related to the direction of $D$ by the Maxwell right-hand rule. Let the symbol $r \uparrow S$ denote the limit in which the point $r$ approaches some point of $S$ moving along the direction of the outward normal vector. Similarly $r \downarrow S$ denotes the limit in which $r$ approaches the same point of $S$ moving in the opposite direction. It will be clear from these definitions that the constrained path integrals $F_{n}\left(r, t \mid r_{i}, t_{i}\right)$ defined by $(2.7 \mathrm{~b})$ are connected on the surface $S$ by the following boundary conditions:

$$
\lim _{r \uparrow S} F_{n}\left(r, t \mid r_{i}, t_{i}\right)=\lim _{r \downarrow S} F_{n+1}\left(r, t \mid r_{i}, t_{i}\right), \quad(r \in S) .
$$


Similar relations hold for all partial derivatives. As all functions $F_{n}$ should be solutions of the Schrödinger equation with a scalar potential, but without the vector potential, the problem is reduced to solving a Schrödinger equation which is much simpler than $(3.1,2)$, but with the unusual boundary conditions (3.4). This method has been used by the author for the case in which $D$ is an infinite straight line, both in the case where $V=0$ and in the case where $V(r)=C_{1} r^{2}+C_{2} r^{2}$ (cf. refs. 13 and 3 ).

The results of the preceding method are in agreement with a calculation by Inomata and $\mathrm{Singh}^{14}$ ) in which the constrained path integral is evaluated directly in polar coordinates. However, the method of these authors seems to be applicable only to the straight line geometry.

The fourth method to evaluate a topologically constrained path integral uses an explicit expression for the entanglement index (Gauss' looping integral, linking number) of $D$ and the electron path $C \equiv\left\{r(t) \mid t_{i}<t<t_{f}\right)$

$$
L[C, D]=(4 \pi)^{1} \oint_{C} \mathrm{~d} \boldsymbol{r} \cdot \oint_{D}|\boldsymbol{r}-\boldsymbol{s}|^{3} \mathrm{~d} s \times(\boldsymbol{r}-s) .
$$

Comparison with (2.3) shows that the entanglement index can be written in terms of the vector potential

$$
L[C, D]=\Phi^{\prime} \oint_{\curlyvee} \boldsymbol{A} \cdot \mathrm{d} r
$$

Hence the constrained path integral can be written in the form

$$
F_{n}(\mathrm{f} \mid \mathrm{i})=\int_{(\mathrm{i})}^{(\mathrm{f})} \delta\left(n-\Phi^{-1} \oint_{i} \boldsymbol{A} \cdot \mathrm{d} \boldsymbol{r}\right) \exp \left\{\frac{\mathrm{i}}{h} \int_{i_{i}}^{t_{i}}\left(\frac{1}{2} m \dot{\boldsymbol{r}}^{2}-V\right) \mathrm{d} t\right\} \mathrm{d}[\boldsymbol{r}(t)],
$$

where the integration now runs over all electron paths connecting (i) and (f). Writing

$$
\delta(x)=\int^{x} \exp (2 \pi k x \mathrm{i}) \mathrm{d} k,
$$

one finds

$$
\begin{aligned}
& F_{n}(\mathrm{f} \mid \mathrm{i})=\int_{-x}^{x} \tilde{F}_{k}(\mathrm{f} \mid \mathrm{i}) \exp (2 \pi n k \mathrm{i}) \mathrm{d} k, \\
& \tilde{F}_{k}(\mathrm{f} \mid \mathrm{i})=\int_{(i)}^{(\mathrm{f})} \exp \left\{\frac{\mathrm{i}}{\hbar} \int_{t_{1}}^{t_{1}}\left(\frac{1}{2} m \dot{r}^{2}-\frac{h k}{\Phi} \boldsymbol{A} \cdot \dot{\boldsymbol{r}}-V\right) \mathrm{d} t\right\} \mathrm{d}[\boldsymbol{r}(t)] .
\end{aligned}
$$


Path integrals of the form (3.10) were studied more than a decade ago by the author (cf. refs. 15 and 16). They can be calculated using a differential equation very similar to, but not identical with, the original Schrödinger equation (3.1). This method has been applied to the infinite straight line geometry ${ }^{4}$ ) and leads to the same results as the three other methods.

Finally, the reader should compare recent work by Brereton and Shah ${ }^{17}$ ) in which certain entanglement problems are recast as a local gauge invariant field theory.

\section{A dilute gas of magnetic flux loops}

In this section we consider the, probably fictitious, case in which space is filled with a dilute gas of microscopic loops of magnetic flux $\Phi$. Suppose loop $k$ has position $\boldsymbol{R}_{k}$ and generates a vector potential $\boldsymbol{A}_{k}$. The total vector potential is

$$
A(r)=\sum_{k} A_{k}(r)
$$

It is straightforward to verify that the considerations in section 2 can be generalized immediately, provided

$$
d \ll \Delta \ll\left|\boldsymbol{r}_{\mathrm{f}}-\boldsymbol{r}_{\mathrm{i}}\right|,
$$

where $d$ denotes the linear dimension of a loop and $\Delta$ the average distance between them. The propagator is given by

$$
G(\mathrm{f} \mid \mathrm{i})=\exp \left(\frac{\mathrm{i} e}{\hbar c} \int_{C_{\mathbf{v}}} \boldsymbol{A} \cdot \mathrm{d} \boldsymbol{r}\right) \sum_{\left\{n_{k}\right\}} F_{\left\{n_{k} \mid\right.}(\mathrm{f} \mid \mathrm{i}) \exp \left(\frac{\mathrm{i} e \Phi}{\hbar c} \sum_{k} n_{k}\right),
$$

in which $F_{\left\{n_{k}\right\}}$ denotes the constrained path integral over all those electron trajectories which connect (i) with (f) and which wind exactly $n_{k}=$ $0, \pm 1, \pm 2, \ldots$ times through the $k$ th flux loop. In the case in which the order of magnitude estimations (4.2) hold true almost all electron paths will wind through a large number of loops; hence, $\Sigma_{k} n_{k} \approx 0$ by mutual cancellation of terms. Also $A \approx 0$ because of the random positions and orientations of the flux loops. Now the propagator will be given to a good approximation by

$$
G(\mathrm{f} \mid \mathrm{i}) \approx \sum_{\left\{n_{k}\right\}} F_{\left\{n_{k}\right\}}(\mathrm{f} \mid \mathrm{i})=G_{0}(\mathrm{f} \mid \mathrm{i}),
$$

which is the free propagator in the absence of any flux loops. In this way one reaches the tentative conclusion that a dilute gas of loops of flux $\Phi$ does not 
lead to an observable (macroscopic) Aharonov-Bohm effect, regardless of the value of $\Phi$, as a result of a mutual cancellation of the Aharonov-Bohm effects of the individual flux loops.

\section{Polymer entanglements}

Exactly solvable models of polymer entanglements have been studied by Prager and Frisch ${ }^{18}$, Edwards ${ }^{19}$, Alexander-Katz and Edwards ${ }^{20}$. Saito and Chen $^{21}$ ), Wiegel ${ }^{13,3}$ ) and Inomata and Singh ${ }^{14}$ ). Since a review can be found in chapter 5 of ${ }^{4}$ ) there is no need to repeat any calculations here. Rather we quote the expression for the configuration sum $Q_{n}\left(\boldsymbol{r}_{N}, N \mid \boldsymbol{r}_{0}, 0\right)$ of a polymer with end points at $\boldsymbol{r}_{N}$ and $\boldsymbol{r}_{0}$, which consists of $N$ repeating units-each represented by a freely hinged link of length $l-$, and which has an entanglement index $n$ with the curve $D$ :

$$
\begin{aligned}
Q_{n}\left(r_{N}, N \mid r_{0}, 0\right)= & \int_{\left(r_{0},(l)\right)}^{\left.r_{N} \cdot N\right)} \delta\left(n-\Phi{ }^{\prime} \oint_{C} \boldsymbol{A} \cdot \mathrm{d} \boldsymbol{r}\right) \exp \left\{-\frac{3}{2 l^{2}} \int_{0}^{N}\left(\frac{\mathrm{d} r}{\mathrm{~d} \nu}\right)^{2} \mathrm{~d} \nu\right. \\
& \left.-\beta \int_{0}^{N} W(\boldsymbol{r}) \mathrm{d} \nu\right\} \mathrm{d}[\boldsymbol{r}(\nu)] .
\end{aligned}
$$

In this equation $\beta=\left(k_{B} T\right)^{\prime}$ and $W$ is the potential of the force per repeating unit. Comparison with (3.7) shows that this problem is in a one-to-one correspondence with the problem of calculating a constrained propagator in the Aharonov-Bohm effect provided the following substitutions are made: $Q_{n}\left(\boldsymbol{r}_{N}, N \mid \boldsymbol{r}_{0}, 0\right) \Leftrightarrow F_{n}\left(\boldsymbol{r}_{\mathrm{f}}, t_{\mathrm{f}} \mid \boldsymbol{r}_{\mathrm{i}}, t_{\mathrm{i}}\right) ; \nu \Leftrightarrow \mathrm{it} / \hbar ; \beta W \Leftrightarrow V ; l^{2} \Leftrightarrow 3 \hbar^{2} / m$. With these substitutions any result about polymer entanglements can be translated in terms of the Aharonov-Bohm effect and inversely.

Some subtle differences between these problems should be noted. In those cases in which the potential $V$ has bound states the Aharonov-Bohm diffraction pattern is determined by the asymptotic behavior of the scattering states. On the other hand, for the polymer entanglement problem the configuration sum (5.1) is dominated for $N \geqslant 1$ by the bound states only. A second striking difference is that a closed polymer can also show self-entanglements, which do occur in electron paths but do not lead to observable effects. These self-entanglement problems cannot be formulated in terms of constrained path integrals but can be broached only with the Alexander polynomial. For this algebraic approach the reader is referred to the papers of Vologodskii et al. ${ }^{22,23}$ ), Frank-Kamenetskii et al. ${ }^{24}$ ) and des Cloizeaux and Mehta $^{25}$ ). 


\section{Acknowledgments}

The author is indebted to Dr. Donald H. Kobe of North Texas State University for discussions on the subject of this paper during our stay at the Aspen Center for Physics during the summer of 1980, and to Dr. W.J. Caspers for critical reading of the manuscript.

\section{References}

1) D.H. Kobe, Ann. Phys. (N.Y.) 123 (1979) 381.

2) C.C. Gerry and Singh V.A., Phys. Rev. D20 (1979) 2550.

3) F.W. Wiegel, Lecture Notes in Physics 121 (Springer, Heidelberg, 1980).

4) F.W. Wiegel, in Phase Transitions and Critical Phenomena, Vol. 8, C. Domb and M.S. Green, eds. (Academic Press, London, to appear).

5) F.B. Fuller, Proc. Nat. Acad. Sci. USA 68 (1971) 815.

6) F.B. Fuller, Proc. Nat. Acad. Sci. USA 75 (1978) 3557.

7) F.H.C. Crick, Proc. Nat. Acad. Sci. USA 73 (1976) 2639.

8) W.F. Pohl and G.W. Roberts, J. Math. Biol. 6 (1978) 383.

9) H. Goldstein, Classical Mechanics (Addison-Wesley, Reading, 1959) p. 21.

10) R.P. Feynman and A.R. Hibbs, Quantum Mechanics and Path Integrals, (McGraw Hill, New York, 1965).

11) L.S. Schulman, J. Math. Phys. 12 (1971) 304.

12) C. Bernido and A. Inomata, Phys. Lett 77A (1980) 394.

13) F.W. Wiegel, J. Chem. Phys. 67 (1977) 469.

14) A. Inomata and V.A. Singh, J. Mat. Phys. 19 (1978) 2318.

15) F.W. Wiegel, Physica 33 (1967) 734.

16) F.W. Wiegel, Physica 37 (1967) 105.

17) M.G. Brereton and S. Shah, J. Phys. A13 (1980) 2751.

18) S. Prager and H.L. Frisch, J. Chem. Phys. 46 (1967) 1475.

19) S.F. Edwards, Proc. Phys. Soc. 91 (1967) 513.

20) R. Alexander-Katz and S.F. Edwards, J. Phys. A5 (1972) 674.

21) N. Saito and Y. Chen, J. Chem. Phys. 59 (1973) 3701.

22) A.V. Vologodskii, A.V. Lukashin, M.D. Frank-Kamenetskii and V.V. Anshelevich, Sov. Phys. JETP 39 (1974) 1059.

23) A.V. Vologodskii, A.V. Lukashin and M.D. Frank-Kamenetskii, Sov. Phys. JETP 40 (1975) 932.

24) M.D. Frank-Kamenetskii, A.V. Lukashin and A.V. Vologodskii, Nature 258 (1975) 398.

25) J. des Cloizeaux and M.L. Mehta, J. Physique 40 (1979) 665. 